\title{
Optimal Control of Cancer Growth
}

\author{
Jens Christian Larsen \\ Vanløse Alle 50 2. mf. tv, 2720 Vanløse, Copenhagen, Denmark \\ Email: jlarsen.math@hotmail.com
}

How to cite this paper: Larsen, J.C. (2019) Optimal Control of Cancer Growth. Applied Mathematics, 10, 173-195.

https://doi.org/10.4236/am.2019.104014

Received: February 15, 2019

Accepted: April 8, 2019

Published: April 11, 2019

Copyright $\odot 2019$ by author(s) and Scientific Research Publishing Inc. This work is licensed under the Creative Commons Attribution International License (CC BY 4.0).

http://creativecommons.org/licenses/by/4.0/

\begin{abstract}
The purpose of the present paper is to apply the Pontryagin Minimum Principle to mathematical models of cancer growth. In [1], I presented a discrete affine model $T$ of cancer growth in the variables $C$ for cancer, $G F$ for growth factors and $G I$ for growth inhibitors. One can sometimes find an affine vector field $X$ on $\mathbb{R}^{3}$ whose time one map is $T$. It is to this vector field we apply the Pontryagin Minimum Principle. We also apply the Discrete Pontryagin Minimum Principle to the model $T$. So we prove that maximal chemo therapy can be optimal and also that it might not depending on the spectral properties of the matrix $A$, (see below). In section five we determine an optimal strategy for chemo or immune therapy.
\end{abstract}

\section{Keywords}

Cancer, Models of Cancer Growth, Pontryagin Minimum Principle

\section{Introduction}

Our reference to optimal control theory of ODEs is [2] and to control theory of discrete systems [3]. There is a review of papers in optimal control theory applied to cancer [4]. The model we consider here is from [1] and is defined by

$$
A=\left(\begin{array}{ccc}
1+\gamma & \alpha & \beta \\
\delta & 1+\mu_{F} & 0 \\
\sigma & 0 & 1+\mu_{I}
\end{array}\right), \quad T(y)=A y+g
$$

where $y=(C, G F, G I)^{\mathrm{T}}, g=\left(g_{C}, g_{F}, g_{I}\right)^{\mathrm{T}} \in \mathbb{R}^{3}$, and $\mathrm{T}$ denotes transpose. Here $\alpha \in \mathbb{R}_{+}, \beta \in \mathbb{R}_{-}, \delta, \sigma \in \mathbb{R}, \mu_{F}, \mu_{I}<0$. We assume that $\alpha \delta+\beta \sigma \neq 0$.

The matrix $A$ has characteristic polynomial $-p(\lambda)$ where

$$
\begin{gathered}
p(\lambda)=\lambda^{3}-\lambda^{2}\left(3+\gamma+\mu_{F}+\mu_{I}\right)-\lambda\left(\alpha \delta+\beta \sigma-\left(1+\mu_{F}\right)\left(1+\mu_{I}\right)\right. \\
-(1+\gamma)\left(2+\mu_{F}+\mu_{I}\right)+\alpha \delta\left(1+\mu_{I}\right)+\beta \sigma\left(1+\mu_{F}\right)-(1+\gamma)\left(1+\mu_{F}\right)\left(1+\mu_{I}\right)
\end{gathered}
$$


and this polynomial can have (i) three real roots or (ii) one real root and two imaginary roots. It turns out that asking $\mu=\mu_{F}=\mu_{I}$ simplifies matters considerably. Then

$$
p(\lambda)=-(1+\mu-\lambda)\left(\lambda^{2}-(2+\gamma+\mu) \lambda+(1+\gamma)(1+\mu)-\alpha \delta-\beta \sigma\right)
$$

So the eigenvalues are $1+\mu$ and

$$
\lambda_{ \pm}=1+\frac{\gamma+\mu}{2} \pm \frac{1}{2} \sqrt{(\gamma-\mu)^{2}+4(\alpha \delta+\beta \sigma)}
$$

We assume that $1+\mu>0, \mu<0$. In case (i) if the eigenvalues of $A, \lambda_{+}, \lambda_{-}, \tilde{\lambda}$ are positive and distinct and we assume this, then you can find an affine vector field $X$ on $\mathbb{R}^{3}$ such that the time one map is $\mathrm{d}$

$$
\Phi_{1}^{X}=T
$$

see [1] and below. In case (ii) if the eigenvalues of $A$ are

$1+\mu, a \pm i b, a>0,1+\mu>0, b \neq 0$ and we assume this, you can also find an affine vector field $X$ on $\mathbb{R}^{3}$ such that

$$
\Phi_{1}^{X}=T
$$

In case (i) define a matrix of eigenvectors

$$
D=\left(\begin{array}{ccc}
1+\mu-\lambda_{+} & 1+\mu-\lambda_{-} & 0 \\
-\delta & -\delta & -\beta \\
-\sigma & -\sigma & \alpha
\end{array}\right)
$$

and in case (ii) define

$$
U=\left(\begin{array}{ccc}
1+\mu-a & -b & 0 \\
-\delta & 0 & -\beta \\
-\sigma & 0 & \alpha
\end{array}\right)
$$

Then in case (i)

$$
\Lambda=D^{-1} A D=\left(\begin{array}{ccc}
\lambda_{+} & 0 & 0 \\
0 & \lambda_{-} & 0 \\
0 & 0 & \tilde{\lambda}
\end{array}\right)
$$

if the eigenvalues are distinct and positive and in case (ii)

$$
\Lambda=U^{-1} A U=\left(\begin{array}{ccc}
a & b & 0 \\
-b & a & 0 \\
0 & 0 & 1+\mu
\end{array}\right)
$$

see [1]. Here $D^{-1}$ denotes the inverse to $D$. To find an $X$ in case (i) define when the eigenvalues are real, positive and distinct, an affine vector field

$$
Y(x)=\left(\begin{array}{ccc}
\ln \lambda_{+} & 0 & 0 \\
0 & \ln \lambda_{-} & 0 \\
0 & 0 & \ln \tilde{\lambda}
\end{array}\right) x+d
$$

Then with the right choice of $d$

$$
\Phi_{1}^{Y}(x)=D^{-1} A D x+D^{-1} g
$$


hence if we let

$$
X(y)=D Y\left(D^{-1}(y)\right)
$$

we get

$$
\Phi_{1}^{X}=T
$$

And to find an $X$ in case (ii) when $a>0, b \neq 0,1+\mu>0$, define

$$
Y(x)=\left(\begin{array}{ccc}
a_{1} & b_{1} & 0 \\
-b_{1} & a_{1} & 0 \\
0 & 0 & \ln (1+\mu)
\end{array}\right)+\tilde{d}
$$

Then with the right choice of $a_{1}, b_{1} \in \mathbb{R}, \tilde{d} \in \mathbb{R}^{3}$ we get

$$
\Phi_{1}^{Y}(x)=U^{-1} A U(x)+U^{-1} g
$$

hence with

$$
X(y)=U Y\left(U^{-1}(y)\right)
$$

we have that the time one map is

$$
\Phi_{1}^{X}(y)=T(y)
$$

See [1] for details of the above and also below.

In section two we solve the problem:

minmize $C(T), T>0$ (fixed) subject to

$$
\left(\begin{array}{c}
C \\
G F \\
G I
\end{array}\right)^{\prime}=X\left(\begin{array}{c}
C \\
G F \\
G I
\end{array}\right)+e_{3} u(t)
$$

$u(t) \in\left[0, g_{I}^{0}\right], g_{I}^{0}>0$, by first solving it for $Y$ and then infer the solution for $X$. Here

$$
e_{3}=B=(0,0,1)^{\mathrm{T}}
$$

and $u(t)$ is piecewise continuous. In section three we apply the discrete Pontryagin minimum principle to the difference equation

$$
x_{k+1}=A x_{k}+B u_{k}+g
$$

where

$$
x_{k}=(C(k), G F(k), G I(k))^{\mathrm{T}}
$$

$u_{k} \in\left[0, g_{I}^{0}\right], g_{I}^{0}>0 . k=0,1, \cdots, N-1$, where $N \in \mathbb{N}, N \geq 2, x_{0}=x$, with the objective to minimize $C(N)$. There are again two cases to consider (i) and (ii) above. If $\mu=\mu_{F}=\mu_{I}$ and the eigenvalues are positive and distinct, maximal chemo therapy is optimal. But in case (ii) it is not always optimal.

When $\mu_{F} \neq \mu_{I}$ and the eigenvalues are real and distinct, we produce a counter example to maximal chemo therapy being optimal, see section four. Some solid tumors grow like Gompertz functions, see [5]. There are several important monographs in mathematics and medicine, see [6]-[11]. [12]-[19] are 
my latest papers on cancer and mathematics. [20] is our reference to roots of cubic polynomials. [21] proves continuous dependence of roots of a polynomial on the coefficients of the polynomial.

In section five we consider optimality of the discrete model $T$ when $\mu_{F} \neq \mu_{I}$. Here we also determine optimal control of the map $T$.

\section{Optimal Control of $X$}

The purpose of this section is to minimize $C(T)$, subject to

$$
\left(\begin{array}{c}
C \\
G F \\
G I
\end{array}\right)^{\prime}=X\left(\begin{array}{c}
C \\
G F \\
G I
\end{array}\right)+e_{3} u(t)
$$

$T>0$ fixed and with $\mu=\mu_{F}=\mu_{I}$. Let us consider (ii) first. We assume that there are a real eigenvalue $1+\mu$ and two imaginary eigenvalues $a \pm i b, a>0,1+\mu>0, b \neq 0$.

Now define the two by two matrix

$$
L_{1}=\left(\begin{array}{cc}
a_{1} & b_{1} \\
-b_{1} & a_{1}
\end{array}\right)
$$

where

$$
\begin{aligned}
& b_{1}=\tan ^{-1}\left(\frac{b}{a}\right) \\
& a_{1}=\ln \sqrt{a^{2}+b^{2}}
\end{aligned}
$$

This will imply, that

$$
\exp \left(\begin{array}{cc}
a_{1} & b_{1} \\
-b_{1} & a_{1}
\end{array}\right)=\left(\begin{array}{cc}
a & b \\
-b & a
\end{array}\right)=L
$$

Also let

$$
\tilde{B}=\left(\begin{array}{ccc}
a_{1} & b_{1} & 0 \\
-b_{1} & a_{1} & 0 \\
0 & 0 & \ln (1+\mu)
\end{array}\right)
$$

and define the vector field

$$
Y(x, v)=\tilde{B} x+d+U^{-1} e_{3} v=\tilde{Y}(x)+U^{-1} e_{3} v
$$

which is affine when $v=0$, where $e_{1}, e_{2}, e_{3}$ is the canonical basis in $\mathbb{R}^{3}$. Also $x, d \in \mathbb{R}^{3}, v \in\left[0, g_{I}^{0}\right], g_{I}^{0}>0$. Put

$$
\tilde{X}(y)=U \tilde{Y} U^{-1}(y)
$$

Let

$$
X(y, v)=\tilde{X}(y)+e_{3} v=U Y\left(U^{-1}(y), v\right)
$$

Define $d_{1}, d_{2}, d_{3}$ by

$$
U^{-1}(g)_{1,2}=L_{1}^{-1}(L-\mathrm{id}) d_{1,2}
$$


and

$$
\frac{\mu}{\ln (1+\mu)} d_{3}=\left(U^{-1} g\right)_{3}
$$

Then

$$
\Phi_{1}^{X}=T
$$

when $v=0$. To this vector field with $v \in\left[0, g_{I}^{0}\right]$ associate the Hamiltonian

$$
H(x, p, v, t)=p^{\mathrm{T}} Y(x, v)
$$

Then we have the adjoint equations

$$
\begin{aligned}
& p_{1}^{\prime}=-\frac{\partial H}{\partial x_{1}}=-p_{1} a_{1}+p_{2} b_{1} \\
& p_{2}^{\prime}=-\frac{\partial H}{\partial x_{2}}=-p_{1} b_{1}-p_{2} a_{1} \\
& p_{3}^{\prime}=-\frac{\partial H}{\partial x_{3}}=-p_{3} \ln (1+\mu)
\end{aligned}
$$

So

$$
p_{1,2}^{\prime}=\left(\begin{array}{cc}
-a_{1} & b_{1} \\
-b_{1} & -a_{1}
\end{array}\right)\left(\begin{array}{l}
p_{1} \\
p_{2}
\end{array}\right)
$$

which has flow

$$
p_{1,2}(t)=\exp \left(-a_{1} t\right)\left(\begin{array}{cc}
\cos \left(b_{1} t\right) & \sin \left(b_{1} t\right) \\
-\sin \left(b_{1} t\right) & \cos \left(b_{1} t\right)
\end{array}\right) p_{1,2}(0)
$$

Define

$$
d(t)=L_{1}^{-1}\left(\exp \left(L_{1} t\right)-\mathrm{id}\right) d_{1,2}
$$

Then the flow of $Y$ is for $v=0$

$$
\begin{gathered}
\Phi^{Y}(t, x)_{1,2}=\exp \left(L_{1} t\right)\left(\begin{array}{l}
x_{1} \\
x_{2}
\end{array}\right)+d(t) \\
\Phi^{Y}(t, x)_{3}=\exp (\ln (1+\mu) t) x_{3}+\frac{\exp (\ln (1+\mu) t)-1}{\ln (1+\mu)} d_{3}
\end{gathered}
$$

Define

$$
S_{1}(x)=(1+\mu-a) x_{1}-b x_{2}
$$

Then we have the transversality conditions

$$
\begin{gathered}
p_{1}(T)=\frac{\partial S_{1}}{\partial x_{1}}=1+\mu-a \\
p_{2}(T)=\frac{\partial S_{1}}{\partial x_{2}}=-b \\
p_{3}(T)=\frac{\partial S_{1}}{\partial x_{3}}=0
\end{gathered}
$$


This means that $p_{3}(t)=0$ and

$$
p_{1,2}(T)=\exp \left(-a_{1} T\right)\left(\begin{array}{cc}
\cos \left(b_{1} T\right) & \sin \left(b_{1} T\right) \\
-\sin \left(b_{1} T\right) & \cos \left(b_{1} T\right)
\end{array}\right) p_{1,2}(0)=\left(\begin{array}{c}
1+\mu-a \\
-b
\end{array}\right)
$$

The two by two matrix in this equation has inverse

$$
\left(\begin{array}{cc}
\cos \left(b_{1} T\right) & -\sin \left(b_{1} T\right) \\
\sin \left(b_{1} T\right) & \cos \left(b_{1} T\right)
\end{array}\right)
$$

So

$$
p_{1,2}(0)=\exp \left(a_{1} T\right)\left(\begin{array}{cc}
\cos \left(b_{1} T\right) & -\sin \left(b_{1} T\right) \\
\sin \left(b_{1} T\right) & \cos \left(b_{1} T\right)
\end{array}\right)\left(\begin{array}{c}
1+\mu-a \\
-b
\end{array}\right)
$$

But then we have

$$
p_{1,2}(t)=\exp \left(a_{1}(T-t)\right)\left(\begin{array}{cc}
\cos \left(b_{1}(t-T)\right) & \sin \left(b_{1}(t-T)\right) \\
-\sin \left(b_{1}(t-T)\right) & \cos \left(b_{1}(t-T)\right)
\end{array}\right)\left(\begin{array}{c}
1+\mu-a \\
-b
\end{array}\right)
$$

Notice that we have

$$
\begin{gathered}
U_{31}=b \beta \\
U_{32}=(1+\mu-a) \beta
\end{gathered}
$$

If

$$
H\left(x^{*}(t), p(t), u^{*}(t), t\right) \leq H\left(x^{*}(t), p(t), u, t\right)
$$

for all $u \in \mathbb{U}=\left[0, g_{I}^{0}\right]$, then $\left(x^{*}(t), u^{*}(t)\right)$ is optimal, see Equations (55) to (60). But this amounts to the inequality

$$
\begin{aligned}
& \left(p_{1}(t) b \beta u_{*}+p_{2}(t)(1+\mu-a) \beta u_{*}\right) \frac{1}{\operatorname{det}(U)} \\
& =\exp \left(a_{1}(T-t)\right)\left(\left(\cos \left(b_{1}(t-T)\right)(1+\mu-a)-b \sin \left(b_{1}(t-T)\right)\right) b \beta u_{*}\right. \\
& \left.+\left(-\sin \left(b_{1}(t-T)\right)(1+\mu-a)-b \cos \left(b_{1}(t-T)\right)\right)\left((1+\mu-a) \beta u_{*}\right)\right) \frac{1}{\operatorname{det}(U)} \\
& =\exp \left(a_{1}(T-t)\right) \sin \left(b_{1}(t-T)\right)\left(-b^{2}-(1+\mu-a)^{2}\right) \beta u_{*} \frac{1}{\operatorname{det}(U)} \\
& =\exp \left(a_{1}(T-t)\right)(\alpha \delta+\beta \sigma) \beta u_{*} \sin \left(b_{1}(t-T)\right) \frac{1}{\operatorname{det}(U)} \\
& \leq \exp \left(a_{1}(T-t)\right) \sin \left(b_{1}(t-T)\right) \frac{\beta u}{-b}
\end{aligned}
$$

where $\operatorname{det}(U)$ denotes the determinant of $U$. We have used that

$$
1+\mu-a=1+\mu-\left(1+\frac{\gamma+\mu}{2}\right)=\frac{\mu-\gamma}{2}
$$

and

$$
\lambda_{+}=1+\frac{\gamma+\mu}{2}+\frac{1}{2} \sqrt{(\gamma-\mu)^{2}+4(\alpha \delta+\beta \sigma)}=a+i b
$$


so that

$$
(1+\mu-a)^{2}+b^{2}=-(\alpha \delta+\beta \sigma)
$$

and also that $\operatorname{det} U=-b(\alpha \delta+\beta \sigma)$. So if

$$
\frac{\sin \left(b_{1}(t-T)\right)}{-b}>0
$$

then

$$
u^{*}(t)=g_{I}^{0}
$$

is optimal and if the reverse inequality holds then

$$
u^{*}(t)=0
$$

is optimal. We shall now consider the case where all eigenvalues $\lambda_{+}, \lambda_{-}, \tilde{\lambda}$ are real, positive and distinct and that $\mu=\mu_{F}=\mu_{I}$. Here we let

$$
Y(x, u)=\tilde{\Lambda} x+d+D^{-1} e_{3} u
$$

where

$$
\tilde{\Lambda}=\operatorname{diag}\left(\ln \lambda_{+}, \ln \lambda_{-}, \ln (1+\mu)\right)
$$

Also define $d$ in

$$
\begin{gathered}
\frac{\lambda_{+}-1}{\ln \lambda_{+}} d_{1}=\left(D^{-1} g\right)_{1} \\
\frac{\lambda_{-}-1}{\ln \lambda_{+}} d_{2}=\left(D^{-1} g\right)_{2} \\
\frac{\mu}{\ln (1+\mu)} d_{3}=\left(D^{-1} g\right)_{3}
\end{gathered}
$$

$d=\left(d_{1}, d_{2}, d_{3}\right)^{\mathrm{T}} \in \mathbb{R}^{3}$. Now define the Hamiltonian

$$
H(x, p, u, t)=p^{\mathrm{T}} Y(x, u)
$$

Then we get the adjoint equations

$$
\begin{gathered}
p_{1}^{\prime}=-\frac{\partial H}{\partial x_{1}}=-\ln \lambda_{+} p_{1} \\
p_{2}^{\prime}=-\frac{\partial H}{\partial x_{2}}=-\ln \lambda_{-} p_{2} \\
p_{3}^{\prime}=-\frac{\partial H}{\partial x_{3}}=-\ln (1+\mu) p_{3}
\end{gathered}
$$

see [2]. Now define

$$
S_{1}(x)=\left(1+\mu-\lambda_{+}\right) x_{1}+\left(1+\mu-\lambda_{-}\right) x_{2}
$$

Then we have the transversality conditions

$$
p(T)=\frac{\partial S_{1}}{\partial x}=\left(\begin{array}{c}
1+\mu-\lambda_{+} \\
1+\mu-\lambda_{-} \\
0
\end{array}\right)
$$


by [2] and see below. Now observe, that

$$
\operatorname{det}(\boldsymbol{D})=\left(\lambda_{+}-\lambda_{-}\right)(\alpha \delta+\beta \sigma)
$$

Because

$$
1+\mu-\lambda_{ \pm}=\frac{\mu-\gamma}{2} \mp \frac{1}{2} \sqrt{(\mu-\gamma)^{2}+4(\alpha \delta+\beta \sigma)}
$$

we find

$$
\left(1+\mu-\lambda_{+}\right)\left(1+\mu-\lambda_{-}\right)=-(\alpha \delta+\beta \sigma)
$$

We shall need

$$
D^{-1}=\left(\begin{array}{ccc}
-\alpha \delta-\beta \sigma & -\alpha\left(1+\mu-\lambda_{-}\right) & -\beta\left(1+\mu-\lambda_{-}\right) \\
\alpha \delta+\beta \sigma & \alpha\left(1+\mu-\lambda_{+}\right) & \beta\left(1+\mu-\lambda_{+}\right) \\
0 & -\sigma\left(\lambda_{+}-\lambda_{-}\right) & \delta\left(\lambda_{+}-\lambda_{-}\right)
\end{array}\right) \frac{1}{\operatorname{det} D}
$$

We have

$$
\left(\begin{array}{c}
C(T) \\
0 \\
0
\end{array}\right)=D\left(\begin{array}{l}
x_{1}(T) \\
x_{2}(T) \\
x_{3}(T)
\end{array}\right)
$$

hence the definition of $S_{1}$. Now

$$
p(T)=\frac{\partial S_{1}}{\partial x}
$$

thus

$$
\begin{aligned}
& p_{1}(T)=\left.p_{1}(0) \exp \left(-\ln \lambda_{+} t\right)\right|_{t=T}=p_{1}(0) \exp \left(-\ln \lambda_{+} T\right)=1+\mu-\lambda_{+} \\
& p_{2}(T)=\left.p_{2}(0) \exp \left(-\ln \lambda_{-} t\right)\right|_{t=T}=p_{2}(0) \exp \left(-\ln \lambda_{-} T\right)=1+\mu-\lambda_{-} \\
& p_{3}(T)=\left.p_{3}(0) \exp (-\ln (1+\mu) t)\right|_{t=T}=p_{3}(0) \exp (-\ln (1+\mu) T)=0
\end{aligned}
$$

So

$$
\begin{gathered}
p_{1}(t)=\left(1+\mu-\lambda_{+}\right) \exp \left(\ln \lambda_{+}(T-t)\right) \\
p_{2}(t)=\left(1+\mu-\lambda_{-}\right) \exp \left(\ln \lambda_{-}(T-t)\right) \\
p_{3}(t)=0
\end{gathered}
$$

If

$$
H\left(x^{*}(t), p(t), u^{*}(t), t\right) \leq H\left(x^{*}(t), p(t), u, t\right)
$$

for all $u \in \mathbb{U},\left(x^{*}(t), u^{*}(t)\right)$ is optimal. And this is equivalent to

$$
p(t)^{\mathrm{T}} D^{-1} e_{3} u^{*}(t) \leq p(t)^{\mathrm{T}} D^{-1} e_{3} u
$$

which again is equivalent to

$$
\begin{aligned}
& \left(1+\mu-\lambda_{+}\right) \exp \left(\ln \lambda_{+}(T-t)\right)(-\beta)\left(1+\mu-\lambda_{-}\right) \frac{u^{*}(t)}{\operatorname{det}(D)} \\
& +\left(1+\mu-\lambda_{-}\right) \exp \left(\ln \lambda_{-}(T-t)\right) \beta\left(1+\mu-\lambda_{+}\right) \frac{u^{*}(t)}{\operatorname{det}(D)}
\end{aligned}
$$




$$
\begin{aligned}
& =u^{*}(t) \beta \frac{-\alpha \delta-\beta \sigma}{\left(\lambda_{+}-\lambda_{-}\right)(\alpha \delta+\beta \sigma)}\left(-\exp \left(\ln \lambda_{+}(T-t)\right)+\exp \left(\ln \lambda_{-}(T-t)\right)\right) \\
& \leq u \beta\left(\exp \left(\ln \lambda_{+}(T-t)\right)-\exp \left(\ln \lambda_{-}(T-t)\right)\right) \frac{1}{\lambda_{+}-\lambda_{-}}
\end{aligned}
$$

for all $u \in \mathbb{U}$. It follows that

$$
u^{*}(t)=g_{I}^{0}
$$

is optimal. We have the two Hamiltonians

$$
\begin{aligned}
& H^{Y}(x, p, u, t)=p^{\mathrm{T}} Y(x, u)=\langle p, Y(x, u)\rangle \\
& H^{X}(y, q, v, t)=q^{\mathrm{T}} X(y, v)=\langle q, X(y, v)\rangle
\end{aligned}
$$

where $\langle\cdot$,$\rangle denotes the canonical inner product. It follows that when the ei-$ genvalues are $a \pm i b, 1+\mu>0, a>0, b \neq 0$

$$
\begin{aligned}
& y(t)=U x(t) \\
& p(t)=U^{\mathrm{T}} q(t)
\end{aligned}
$$

if

$$
\begin{gathered}
y(0)=U x(0) \\
p(T)=U^{\mathrm{T}} q(T)
\end{gathered}
$$

where $y(t)$ is an integral curve of $X$ and $x(t)$ an integral curve of $Y$. Because

$$
q^{\prime}=-\frac{\partial H^{X}}{\partial y}=-\left(U^{-1}\right)^{\mathrm{T}} \tilde{B}^{\mathrm{T}} U^{\mathrm{T}} q
$$

hence

$$
U^{\mathrm{T}} q^{\prime}=-\tilde{B}^{\mathrm{T}} U^{\mathrm{T}} q
$$

and

$$
p^{\prime}=-\tilde{B}^{\mathrm{T}} p
$$

We now get, that

$$
\begin{aligned}
H^{X}(y(t), q(t), v(t), t) & =\langle q(t), X(y(t), v(t))\rangle \\
& =\left\langle q(t), U Y\left(U^{-1} y(t), v(t)\right)\right\rangle \\
& =\left\langle U^{\mathrm{T}}(q(t)), Y\left(U^{-1}(y(t)), v(t)\right)\right\rangle \\
& =\langle p(t), Y(x(t), v(t))\rangle \\
& =H^{Y}(x(t), p(t), v(t), t)
\end{aligned}
$$

So

$$
H^{X}(y(t), q(t), v(t), t)=H^{Y}(x(t), p(t), v(t), t)
$$

When the eigenvalues of $A$ are real, distinct and positive

$$
y(t)=D x(t)
$$




$$
p(t)=D^{\mathrm{T}} q(t)
$$

if

$$
\begin{gathered}
y(0)=D x(0) \\
p(T)=D^{\mathrm{T}} q(T)
\end{gathered}
$$

We also have

$$
q^{\prime}=-\left(D^{-1}\right)^{\mathrm{T}} \Lambda^{\mathrm{T}} D^{\mathrm{T}} q
$$

and

$$
\left(D^{\mathrm{T}} q\right)^{\prime}=-\Lambda^{\mathrm{T}} D^{\mathrm{T}} q
$$

thus

$$
p^{\prime}=-\Lambda^{\mathrm{T}} p
$$

Hence

$$
H^{X}(y(t), q(t), v(t), t)=H^{Y}(x(t), p(t), v(t), t)
$$

We need the following theorem, which is well known.

Theorem 1 Now The following statements about a $C^{2}$ function from

$$
f: \mathbb{R}^{n} \rightarrow \mathbb{R}
$$

to the reals, where $n$ is a positive integer, $n \in \mathbb{N}$ are equivalent:

(i) $f(\lambda x+(1-\lambda) y) \leq \lambda f(x)+(1-\lambda) f(y)$ where $\lambda \in[0,1]$;

(ii) $f(y) \geq f(x)+\frac{\partial f}{\partial x}(x)(y-x)$;

(iii) $\sum_{i, j=1, \cdots, n} \gamma_{i} \frac{\partial^{2} f}{\partial x_{i} \partial x_{j}} \gamma_{j} \geq 0$

where $x, y, \gamma \in \mathbb{R}^{n}$.

$(x(t), u(t))$ is admissible by definition if $0 \leq u(t) \leq g_{I}^{0}$ and

$$
x^{\prime}(t)=Y(x(t), u(t)), x(0)=x_{0} \in \mathbb{R}^{3}
$$

To see that

$$
S_{1}\left(x^{*}(T)\right) \leq S_{1}(x(T))
$$

for $\left(x^{*}(t), u^{*}(t)\right)$ optimal candidate and $(x(t), u(t))$ admissible argue as in [2]

$$
\begin{aligned}
\Delta & =S_{1}\left(x^{*}(T)\right)-S_{1}(x(T)) \\
& =S_{1}\left(x^{*}(T)\right)-S_{1}(x(T)) \\
& +\int_{0}^{T}\left(H^{Y}\left(x^{*}(t), p(t), u^{*}(t), t\right)-p(t)^{\mathrm{T}} x^{* \prime}(t)\right) \\
& -\left(H^{Y}(x(t), p(t), u(t), t)-p(t)^{\mathrm{T}} x^{\prime}(t)\right) \mathrm{d} t
\end{aligned}
$$

We have the following inequality, which follows from theorem 1. 


$$
\begin{aligned}
& H^{Y}\left(x^{*}, p, u^{*}, t\right)-H^{Y}(x, p, u, t) \\
& \leq \frac{\partial H^{Y}}{\partial x}\left(x^{*}, p, u^{*}, t\right)\left(x^{*}-x\right)+\frac{\partial H^{Y}}{\partial u}\left(x^{*}, p, u^{*}, t\right)\left(u^{*}-u\right)
\end{aligned}
$$

We also have

$$
p^{\prime}=-\frac{\partial H^{Y}}{\partial x}\left(x^{*}, p, u^{*}, t\right)
$$

We can now estimate

$$
\begin{aligned}
\Delta \leq & S_{1}\left(x^{*}(T)\right)-S_{1}(x(T))+\int_{0}^{T} p^{\prime \mathrm{T}}\left(x-x^{*}\right)+p^{\mathrm{T}}\left(x^{\prime}-x^{* \prime}\right) \mathrm{d} t \\
& +\int_{0}^{T} \frac{\partial H^{Y}}{\partial u}\left(x^{*}, p, u^{*}, t\right)\left(u^{*}-u\right) \mathrm{d} t \\
& \leq \int_{0}^{T} \frac{\mathrm{d}}{\mathrm{d} t}\left(p^{\mathrm{T}}\left(x-x^{*}\right)\right)(t) \mathrm{d} t+S_{1}\left(x^{*}(T)\right)-S_{1}(x(T)) \\
& =p(T)^{\mathrm{T}}\left(x(T)-x^{*}(T)\right)+S_{1}\left(x^{*}(T)\right)-S_{1}(x(T)) \\
& =\frac{\partial S_{1}}{\partial x}\left(x^{*}(T)\right)\left(x(T)-x^{*}(T)\right)+S_{1}\left(x^{*}(T)\right)-S_{1}(x(T)) \\
& \leq 0
\end{aligned}
$$

because $S_{1}$ is convex, by theorem 1 . We have used, that we have arranged, that

$$
\frac{\partial H}{\partial u}\left(x^{*}, p, u^{*}, t\right)\left(u^{*}-u\right) \leq 0
$$

for all $u \in \mathbb{U}$, by the mean value theorem.

We have optimality.

\section{Optimal Control of $T$}

In this section we consider the problem: minimize $C(N)$ subject to

$$
y_{k+1}=A y_{k}+B u_{k}+g=f\left(y_{k}, u_{k}\right)
$$

$k=1, \cdots, N-1, N \in \mathbb{N}, N \geq 2, u(k) \in \mathbb{U}=\left[0, g_{I}^{0}\right], g_{i}^{0}>0, \mu=\mu_{F}=\mu_{I}$ where $A$ is as in the introduction.

Also

$$
y_{k}=(C(k), G F(k), G I(k)), g \in \mathbb{R}^{3}
$$

Here

$$
B=(0,0,1)^{\mathrm{T}}, \quad g=\left(g_{C}, g_{F}, g_{I}\right)^{\mathrm{T}}
$$

Assume (i) the eigenvalues of $A$ are real and distinct.

In the Discrete Pontryagin Minimal Principle applied to $T$ you define the Hamiltonian by (138) and then you find

$$
\frac{\partial H}{\partial u_{k}}\left(x_{k}^{*}, u_{k}^{*}, \lambda_{k}\right)\left(u_{k}^{*}-u_{k}\right)
$$

and minimize it to find the optimal control $u_{k}^{*}$. It is optimal due to computations (157) to (163) below. 
Define then the Hamiltonian

$$
H\left(x_{k}, u_{k}, \lambda_{k}\right)=\lambda_{k}^{\mathrm{T}}\left(\tilde{A} x_{k}+\hat{B} u_{k}+D^{-1} g\right)
$$

where $\lambda_{k} \in \mathbb{R}^{3}$ and

$$
\begin{gathered}
\hat{B}=D^{-1} B \\
\tilde{A}=D^{-1} A D
\end{gathered}
$$

Then we have the adjoint equation

$$
\lambda_{k-1}=\frac{\partial H}{\partial x_{k}}\left(x_{k}, u_{k}, \lambda_{k}\right)=\tilde{A}^{\mathrm{T}} \lambda_{k}
$$

Inductively

$$
\lambda_{N-k-1}=\left(\tilde{A}^{\mathrm{T}}\right)^{k} \lambda_{N-1}
$$

In particular

$$
\lambda_{0}=\left(\tilde{A}^{\mathrm{T}}\right)^{N-1} \lambda_{N-1}
$$

For $k=0$ we have

$$
\begin{aligned}
& H\left(x_{0}^{*}, u_{0}^{*}, \lambda_{0}\right)=\left(\lambda_{0}\right)^{\mathrm{T}}\left(\tilde{A} x_{0}^{*}+\hat{B} u_{0}^{*}+D^{-1} g\right) \\
& \leq H\left(x_{0}^{*}, u, \lambda_{0}\right)=\left(\lambda_{0}\right)^{\mathrm{T}}\left(\tilde{A} x_{0}^{*}+\hat{B} u+D^{-1} g\right)
\end{aligned}
$$

which is equivalent to

$$
\left(\lambda_{0}\right)^{\mathrm{T}} \hat{B} u_{0}^{*} \leq\left(\lambda_{0}\right)^{\mathrm{T}} \hat{B} u
$$

Define

$$
S_{1}(x)=F(x)=\left(1+\mu-\lambda_{+}\right) x_{1}+\left(1+\mu-\lambda_{-}\right) x_{2}
$$

Now

$$
\lambda_{0}=\left(\tilde{A}^{\mathrm{T}}\right)^{N-1} \lambda_{N-1}
$$

where

$$
\lambda_{N-1}=\frac{\partial F}{\partial x}\left(x_{N}\right)
$$

Thus

$$
\left(\lambda_{0}\right)^{\mathrm{T}} \hat{B} u_{0}^{*}=\left(\left(\tilde{A}^{\mathrm{T}}\right)^{N-1} \lambda_{N-1}\right)^{\mathrm{T}} \hat{B} u_{0}^{*}=\left(\lambda_{N-1}\right)^{\mathrm{T}} \tilde{A}^{N-1} \hat{B} u_{0}^{*}=\frac{\partial F^{\mathrm{T}}}{\partial x}\left(x_{N}\right) \tilde{A}^{N-1} \hat{B} u_{0}^{*}
$$

Assume that (i) holds and $\lambda_{+}, \lambda_{-}, 1+\mu \in \mathbb{R}$ are distinct, when $\mu=\mu_{F}=\mu_{I}$. Then

$$
\hat{B}=\left(\begin{array}{c}
D_{31} \\
D_{32} \\
D_{33}
\end{array}\right) \frac{1}{\operatorname{det}(D)}=\left(\begin{array}{c}
-\beta\left(1+\mu-\lambda_{-}\right) \\
\beta\left(1+\mu-\lambda_{+}\right) \\
\delta\left(\lambda_{+}-\lambda_{-}\right)
\end{array}\right) \frac{1}{\operatorname{det}(D)}=D^{-1} e_{3}
$$

So now we get, that (146) amounts to 


$$
\begin{gathered}
\left(1+\mu-\lambda_{+}, 1-\mu-\lambda_{-}, 0\right)\left(\begin{array}{ccc}
\lambda_{+}^{N-1} & 0 & 0 \\
0 & \lambda_{-}^{N-1} & 0 \\
0 & 0 & (1+\mu)^{N-1}
\end{array}\right) \hat{B} \\
=\frac{-\beta(-\alpha \delta-\beta \sigma) \lambda_{+}^{N-1}}{\operatorname{det}(D)}+\frac{\beta(-\alpha \delta-\beta \sigma) \lambda_{-}^{N-1}}{\operatorname{det}(D)} \\
=\frac{\alpha \delta+\beta \sigma}{\left(\lambda_{+}-\lambda_{-}\right)(\alpha \delta+\beta \sigma)} \beta\left(\lambda_{+}^{N-1}-\lambda_{-}^{N-1}\right) \\
=\frac{\beta}{\lambda_{+}-\lambda_{-}}\left(\lambda_{+}^{N-1}-\lambda_{-}^{N-1}\right) \leq 0
\end{gathered}
$$

Similarly for $k=1, \cdots, N-2$

$$
\lambda_{k}^{\mathrm{T}} \hat{B} u_{k}^{*}=\left(1+\mu-\lambda_{+}, 1+\mu-\lambda_{-}, 0\right)\left(\begin{array}{ccc}
\lambda_{+}^{N-k-1} & 0 & 0 \\
0 & \lambda_{-}^{N-k-1} & 0 \\
0 & 0 & (1+\mu)^{N-k-1}
\end{array}\right) \hat{B} u_{k}^{*}<0
$$

For $k=N-1$ we have, that $(1,0,0) \cdot \hat{B}=0$. This means that maximal chemo therapy is optimal. Because similar to (128) to (132), we get

$$
\begin{aligned}
& \Delta=S_{1}\left(x_{N}^{*}\right)-S_{1}\left(x_{N}\right) \\
& =S_{1}\left(x_{N}^{*}\right)-S_{1}\left(x_{N}\right)+\sum_{k=0}^{N-1}\left(H\left(x_{k}^{*}, u_{k}^{*}, \lambda_{k}\right)-H\left(x_{k}, u_{k}, \lambda_{k}\right)-\lambda_{k}^{\mathrm{T}} x_{k+1}^{*}+\lambda_{k}^{\mathrm{T}} x_{k+1}\right) \\
& \leq S_{1}\left(x_{N}^{*}\right)-S_{1}\left(x_{N}\right)+\sum_{k=0}^{N-1} \frac{\partial H}{\partial x_{k}}\left(x_{k}^{*}, u_{k}^{*}, \lambda_{k}\right)\left(x_{k}^{*}-x_{k}\right) \\
& +\frac{\partial H}{\partial u_{k}}\left(x_{k}^{*}, u_{k}^{*}, \lambda_{k}\right)\left(u_{k}^{*}-u_{k}\right)-\lambda_{k}^{\mathrm{T}} x_{k+1}^{*}+\lambda_{k}^{\mathrm{T}} x_{k+1} \\
& \quad \leq S_{1}\left(x_{N}^{*}\right)-S_{1}\left(x_{N}\right)+\sum_{k=0}^{N-1} \lambda_{k-1}^{\mathrm{T}}\left(x_{k}^{*}-x_{k}\right)-\lambda_{k}^{\mathrm{T}} x_{k+1}^{*}+\lambda_{k}^{\mathrm{T}} x_{k+1} \\
& =S_{1}\left(x_{N}^{*}\right)-S_{1}\left(x_{N}\right)-\lambda_{N-1}^{\mathrm{T}}\left(x_{N}^{*}-x_{N}\right) \\
& =S_{1}\left(x_{N}^{*}\right)-S_{1}\left(x_{N}\right)-\frac{\partial S_{1}}{\partial x}\left(x_{N}^{*}\right)\left(x_{N}^{*}-x_{N}\right) \leq 0
\end{aligned}
$$

by theorem 1 and since $S_{1}$ is convex.

Then we have

$$
C_{*}(N)-C(N)=S_{1}\left(x_{N}^{*}\right)-S_{1}\left(x_{N}\right) \leq 0
$$

As above we get

$$
\tilde{H}\left(y_{k}, u_{k}, \zeta_{k}\right)=\zeta_{k}^{\mathrm{T}}\left(A y_{k}+g+e_{3} v_{k}\right)
$$

and

$$
y_{k+1}=A y_{k}+g+e_{3} v_{k}
$$

Also

$$
\zeta_{k-1}=A^{\mathrm{T}} \zeta_{k}
$$


and

$$
\lambda_{k-1}=\tilde{A}^{\mathrm{T}} \lambda_{k}
$$

Thus

$$
\lambda_{k-1}=D^{\mathrm{T}} \zeta_{k-1}
$$

When

$$
y_{0}=D x_{0}
$$

then

$$
y_{k}=D x_{k}
$$

Hence

$$
\tilde{H}\left(y_{k}, v_{k}, \zeta_{k}\right)=H\left(x_{k}, v_{k}, \lambda_{k}\right)
$$

Now consider the case where there are imaginary eigenvalues $a \pm i b, a>0, b \neq 0,1+\mu>0$ for $A$. We need the following well known formulas for

$$
\left(\begin{array}{cc}
a & b \\
-b & a
\end{array}\right)^{2 p+1}=\left(\begin{array}{cc}
A_{p} & B_{p} \\
-B_{p} & A_{p}
\end{array}\right)
$$

which are well known, where $p \in \mathbb{N}_{0}$ and

$$
\begin{aligned}
& A_{p}=\sum_{q=0}^{p}\left(\begin{array}{c}
2 p+1 \\
2 q
\end{array}\right)(-1)^{q} b^{2 q} a^{2 p+1-2 q} \\
& B_{p}=\sum_{q=0}^{p}\left(\begin{array}{c}
2 p+1 \\
2 q+1
\end{array}\right)(-1)^{q} b^{2 q+1} a^{2 p-2 q}
\end{aligned}
$$

and $p \in \mathbb{N}$

$$
\left(\begin{array}{cc}
a & b \\
-b & a
\end{array}\right)^{2 p}=\left(\begin{array}{cc}
C_{p} & D_{p} \\
-D_{p} & C_{p}
\end{array}\right)
$$

where

$$
\begin{gathered}
C_{p}=\sum_{q=0}^{p}\left(\begin{array}{c}
2 p \\
2 q
\end{array}\right)(-1)^{q} b^{2 q} a^{2 p-2 q} \\
D_{p}=\sum_{q=0}^{p-1}\left(\begin{array}{c}
2 p \\
2 q+1
\end{array}\right)(-1)^{q} b^{2 q+1} a^{2 p-1-2 q}
\end{gathered}
$$

You can prove them by induction. We have

$$
\begin{aligned}
& H\left(x_{k}, v_{k}, \lambda_{k}\right)=\left\langle\lambda_{k}, \tilde{B} x_{k}+U^{-1} e_{3} v_{k}+U^{-1} g\right\rangle \\
& =\tilde{H}\left(y_{k}, v_{k}, \zeta_{k}\right)=\left\langle\zeta_{k}, A y_{k}+g+e_{3} v_{k}\right\rangle
\end{aligned}
$$

where

$$
\tilde{B}=\left(\begin{array}{ccc}
a & b & 0 \\
-b & a & 0 \\
0 & 0 & 1+\mu
\end{array}\right)
$$

As above 


$$
\lambda_{k}=\left(\tilde{B}^{\mathrm{T}}\right)^{N-k-1} \lambda_{N-1}=\left(\tilde{B}^{\mathrm{T}}\right)^{N-k-1} \frac{\partial F}{\partial x}
$$

where

$$
S_{1}(x)=F(x)=(1+\mu-a) x_{1}-b x_{2}
$$

So

$$
\lambda_{k}^{\mathrm{T}} \hat{B}=(1+\mu-a,-b, 0) \tilde{B}^{N-k-1}\left(\beta b, \beta(1+\mu-a),{ }^{*}\right)^{\mathrm{T}} \frac{1}{\operatorname{det}(U)}
$$

For $k \geq 0$ we find when $N-k-1=2 p+1, k \neq N-1$

$$
\begin{aligned}
\frac{\partial H}{\partial v_{k}} & =(1+\mu-a,-b)\left(\begin{array}{cc}
A_{p} & B_{p} \\
-B_{p} & A_{p}
\end{array}\right) \beta\left(\begin{array}{c}
b \\
1+\mu-a
\end{array}\right) \frac{1}{\operatorname{det}(U)} \\
& =\beta\left((1+\mu-a)\left(A_{p} b+(1+\mu-a) B_{p}\right)-b\left(-B_{p} b+(1+\mu-a) A_{p}\right)\right) \frac{1}{\operatorname{det}(U)} \\
& =\beta\left((1+\mu-a)^{2}+b^{2}\right) B_{p} \frac{1}{\operatorname{det}(U)} \\
& =\beta(-\alpha \delta-\beta \sigma) B_{p} \frac{1}{\operatorname{det}(U)} \\
& =\frac{\beta}{b} B_{p}
\end{aligned}
$$

Here $\operatorname{det}(U)=-b(\alpha \delta+\beta \sigma)$. When $N-k-1=2 p, k \neq N-1$

$$
\frac{\partial H}{\partial v_{k}}=(1+\mu-a,-b)\left(\begin{array}{cc}
C_{p} & D_{p} \\
-D_{p} & C_{p}
\end{array}\right) \beta\left(\begin{array}{c}
b \\
1+\mu-a
\end{array}\right) \frac{1}{\operatorname{det}(U)}=\frac{\beta}{b} D_{p}
$$

If

$$
\frac{\beta}{b} B_{p}<0
$$

let

$$
v_{k}^{*}=g_{I}^{0}
$$

and

$$
v_{k}^{*}=0
$$

if the reverse inequality holds. If

$$
\frac{\beta}{b} D_{p}<0
$$

let

$$
v_{k}^{*}=g_{I}^{0}
$$

and

$$
v_{k}^{*}=0
$$

if the reverse inequality holds. Then $\left(x_{k}^{*}, v_{k}^{*}\right)$ is optimal, by (157) to (163). For 
$k=N-1$ we have $(1,0,0) \cdot B=0$.

\section{A Counter Example}

We shall now present a counter example to optimality of maximal chemo therapy when the eigenvalues are real and $\mu_{F} \neq \mu_{I}$ for the model

$$
T(y)=A y+g
$$

of the introduction.

Remember the definition of the discriminant $\Delta$ of a cubic polynomial

$$
p(\lambda)=\lambda^{3}+a_{1} \lambda^{2}+a_{2} \lambda+a_{3}
$$

Namely

$$
-108 \Delta=a_{1}^{2} a_{2}^{2}-27 a_{3}^{2}-4 a_{2}^{3}-4 a_{1}^{3} a_{3}+18 a_{1} a_{2} a_{3}
$$

Notice that the degree of $1+\gamma$ is four in $a_{1}^{2} a_{2}^{2}$ and $-4 a_{1}^{3} a_{3}$ while it is only two or three in $-27 a_{3}^{2},-4 a_{2}^{3}, 18 a_{1} a_{2} a_{3}$. We thus get

$-108 \Delta=a_{1}^{2} a_{2}^{2}-4 a_{1}^{3} a_{3}+$ lower order terms in $(1+\gamma)$

$$
\begin{aligned}
& =\left(3+\gamma+\mu_{F}+\mu_{I}\right)^{2}(\alpha \delta+\beta \sigma)-\left(1+\mu_{F}\right)\left(1+\mu_{I}\right)-(1+\gamma)\left(2+\mu_{F}+\mu_{I}\right)^{2} \\
& +4\left(3+\gamma+\mu_{F}+\mu_{I}\right)^{3}\left(\alpha \delta\left(1+\mu_{I}\right)+\beta \sigma\left(1+\mu_{F}\right)-(1+\gamma)\left(1+\mu_{F}\right)\left(1+\mu_{I}\right)\right) \\
& + \text { lower order terms in }(1+\gamma)
\end{aligned}
$$

which becomes

$$
\begin{aligned}
& \left((1+\gamma)^{2}+\left(1+\mu_{F}\right)^{2}+\left(1+\mu_{I}\right)^{2}+2(1+\gamma)\left(1+\mu_{F}\right)+2(1+\gamma)\left(1+\mu_{I}\right)\right. \\
\left.+2\left(1+\mu_{F}\right)\left(1+\mu_{I}\right)\right)\left(\alpha \delta+\beta \sigma-\left(1+\mu_{F}\right)\left(1+\mu_{I}\right)-(1+\gamma)\left(2+\mu_{F}+\mu_{I}\right)\right)^{2} & \\
+ & 4\left((1+\gamma)^{3}+\text { lower order terms in }(1+\gamma)\right)\left(\alpha \delta\left(1+\mu_{I}\right)+\beta \sigma\left(1+\mu_{F}\right)\right. \\
& \left.-(1+\gamma)\left(1+\mu_{F}\right)\left(1+\mu_{I}\right)\right) \\
= & (1+\gamma)^{2}(1+\gamma)^{2}\left(1+\mu_{F}+1+\mu_{I}\right)^{2}-4(1+\gamma)^{4}\left(1+\mu_{F}\right)\left(1+\mu_{I}\right) \\
& + \text { lower order terms in }(1+\gamma)
\end{aligned}
$$

and this is

$$
\begin{aligned}
& (1+\gamma)^{4}\left(\left(1+\mu_{F}\right)^{2}+\left(1+\mu_{I}\right)^{2}+2\left(1+\mu_{F}\right)\left(1+\mu_{I}\right)-4\left(1+\mu_{F}\right)\left(1+\mu_{I}\right)\right) \\
& + \text { lower order terms in }(1+\gamma) \\
& =(1+\gamma)^{4}\left(\mu_{F}-\mu_{I}\right)^{2}+\text { lower order terms in }(1+\gamma)
\end{aligned}
$$

It follows that for $-\gamma$ large

$$
\Delta<0
$$

so that there are three real distinct roots, see Uspensky [20]. But

$$
A_{13}^{2}=\beta(1+\gamma)+\beta\left(1+\mu_{I}\right)>0
$$

when $-\gamma$ is large. So maximal chemo therapy is not optimal for $N=3$. In fact 


$$
x_{3}=A^{3} x_{0}+A^{2}\left(B u_{0}+g\right)+A\left(B u_{1}+g\right)+B u_{2}+g
$$

So $u_{0}=0, u_{1}=g_{I}^{0}$ gives an optimal trajectory.

\section{Optimality of $T$ When $\mu_{F} \neq \mu_{I}$}

Consider the model $T$ from the introduction

$$
T(y)=A y+g+u e_{3}
$$

where

$$
A=\left(\begin{array}{ccc}
1+\gamma & \alpha & \beta \\
\delta & 1+\mu_{F} & 0 \\
\sigma & 0 & 1+\mu_{I}
\end{array}\right)
$$

and

$$
y=(C, G F, G I)^{\mathrm{T}}, \quad g=\left(g_{C}, g_{F}, g_{I}\right)^{\mathrm{T}} \in \mathbb{R}^{3}
$$

Assume (i) the eigenvalues $\lambda_{+}, \lambda_{-}, \tilde{\lambda}=1+\mu$ of $A$ are real and distinct, when $\mu=\mu_{F}=\mu_{I}$.

Theorem 2 There exists a Euclidean open ball $B_{\rho}(\mu, \mu), \rho>0$ in $\mathbb{R}^{2}$, such that for $\left(\mu_{F}, \mu_{I}\right) \in B_{\rho}(\mu, \mu)$ maximal chemo therapy is optimal.

Also let

$$
D=\left(\begin{array}{ccc}
\left(1+\mu_{F}-\lambda_{+}\right)\left(1+\mu_{I}-\lambda_{+}\right) & \left(1+\mu_{F}-\lambda_{-}\right)\left(1+\mu_{I}-\lambda_{-}\right) & -\beta\left(1+\mu_{F}-\tilde{\lambda}\right) \\
-\delta\left(1+\mu_{I}-\lambda_{+}\right) & -\delta\left(1+\mu_{I}-\lambda_{-}\right) & \delta \beta \\
-\sigma\left(1+\mu_{F}-\lambda_{+}\right) & -\sigma\left(1+\mu_{F}-\lambda_{-}\right) & -\alpha \delta+(1+\gamma-\tilde{\lambda})\left(1+\mu_{F}-\tilde{\lambda}\right)
\end{array}\right)
$$

be a matrix with column eigenvectors to the eigenvalues $\lambda_{+}, \lambda_{-}, \tilde{\lambda}$ of $A$. We have $(\delta \neq 0)$

$$
\begin{aligned}
\left.\operatorname{det}(D)\right|_{\mu_{F}=\mu_{I}=\mu}= & \left(1+\mu-\lambda_{+}\right)\left(1+\mu-\lambda_{-}\right)\left|\begin{array}{ccc}
1+\mu-\lambda_{+} & 1+\mu-\lambda_{-} & 0 \\
-\delta & -\delta & \beta \delta \\
-\sigma & -\sigma & -\alpha \delta
\end{array}\right| \\
= & \left(1+\mu-\lambda_{+}\right)\left(\delta^{2} \alpha+\beta \sigma \delta\right)(-\alpha \delta-\sigma \beta) \\
& -\left(1+\mu-\lambda_{-}\right)\left(\delta^{2} \alpha+\beta \sigma \delta\right)(-\alpha \delta-\sigma \beta) \\
= & \left(\lambda_{+}-\lambda_{-}\right)(\alpha \delta+\beta \sigma)^{2} \delta
\end{aligned}
$$

Define the Hamiltonian

$$
\begin{gathered}
H\left(x_{k}, u_{k}, \lambda_{k}\right)=\lambda_{k}^{\mathrm{T}} f_{k}\left(x_{k}, u_{k}\right)=\lambda_{k}^{\mathrm{T}}\left(A x_{k}+g+B u_{k}\right) \\
k=0, \cdots, N-1 \text { and } \\
F(x)=\left(1+\mu_{F}-\lambda_{+}\right)\left(1+\mu_{I}-\lambda_{+}\right) x_{1}+\left(1+\mu_{F}-\lambda_{-}\right)\left(1+\mu_{I}-\lambda_{-}\right) x_{2} \\
-\beta\left(1+\mu_{F}-\tilde{\lambda}\right) x_{3}
\end{gathered}
$$

We have

$$
\lambda_{N-k-1}=\left(A^{\mathrm{T}}\right)^{k} \lambda_{N-1}
$$


In particular

$$
\lambda_{0}=\left(A^{\mathrm{T}}\right)^{N-1} \lambda_{N-1}=\left(A^{\mathrm{T}}\right)^{N-1} \frac{\partial F}{\partial x}\left(x_{N}^{*}\right)
$$

Now consider the system

$$
y_{k+1}=D^{-1} A D y_{k}+D^{-1} g+D^{-1} e_{3} u_{k}
$$

which is conjugate to the $x_{k}$ system. Now observe that with

$$
\tilde{H}\left(y_{k}, v_{k}, \zeta_{k}\right)=\zeta_{k}^{\mathrm{T}}\left(\Lambda y_{k}+D^{-1} g+D^{-1} e_{3} v_{k}\right)
$$

and

$$
\Lambda=D^{-1} A D
$$

we have that

$$
\zeta_{k}=\left(\Lambda^{\mathrm{T}}\right)^{N-k-1} \zeta_{N-1}
$$

and

$$
\begin{gathered}
\zeta_{0}=\left(\Lambda^{\mathrm{T}}\right)^{N-1} \zeta_{N-1} \\
\tilde{H}\left(y_{0}^{*}, v_{0}^{*}, \zeta_{k}\right) \leq \tilde{H}\left(y_{0}^{*}, v_{0}, \zeta_{0}\right)
\end{gathered}
$$

is equivalent to

$$
\zeta_{0}^{\mathrm{T}} D^{-1} B v_{0}^{*} \leq \zeta_{0}^{\mathrm{T}} D^{-1} B v_{0}
$$

So if

$$
\Delta=\zeta_{0}^{\mathrm{T}} D^{-1} B<0
$$

then maximal chemo therapy is optimal, by a computation like (157) to (163). Here

$$
\begin{aligned}
& \left(\zeta_{N-1}\right)^{\mathrm{T}}=\frac{\partial F}{\partial x}\left(x_{N}^{*}\right)^{\mathrm{T}} \\
& =\left(\left(1+\mu_{F}-\lambda_{+}\right)\left(1+\mu_{I}-\lambda_{+}\right),\left(1+\mu_{F}-\lambda_{-}\right)\left(1+\mu_{I}-\lambda_{-}\right),-\beta\left(1+\mu_{F}-\tilde{\lambda}\right)\right)
\end{aligned}
$$

But this amounts to

$$
\begin{aligned}
\Delta= & \left(1+\mu_{F}-\lambda_{+}\right)\left(1+\mu_{I}-\lambda_{+}\right) \lambda_{+}^{N-1} \frac{D_{31}}{\operatorname{det}(D)} \\
& +\left(1+\mu_{F}-\lambda_{-}\right)\left(1+\mu_{I}-\lambda_{-}\right) \lambda_{-}^{N-1} \frac{D_{32}}{\operatorname{det}(D)} \\
& -\beta\left(1+\mu_{F}-\tilde{\lambda}\right) \tilde{\lambda}^{N-1} \frac{D_{33}}{\operatorname{det}(D)}
\end{aligned}
$$

where $D_{i j}$ are complements in $D$. Hence

$$
\begin{gathered}
D_{31}=\left(1+\mu_{F}-\lambda_{-}\right)\left(1+\mu_{I}-\lambda_{-}\right) \delta \beta-\delta \beta\left(1+\mu_{I}-\lambda_{-}\right)\left(1+\mu_{F}-\tilde{\lambda}\right) \\
D_{32}=-\left(1+\mu_{F}-\lambda_{+}\right)\left(1+\mu_{I}-\lambda_{+}\right) \delta \beta+\delta \beta\left(1+\mu_{I}-\lambda_{+}\right)\left(1+\mu_{F}-\tilde{\lambda}\right) \\
D_{33}=\left(1+\mu_{F}-\lambda_{+}\right)\left(1+\mu_{I}-\lambda_{+}\right)(-\delta)\left(1+\mu_{I}-\lambda_{-}\right) \\
+\delta\left(1+\mu_{I}-\lambda_{+}\right)\left(1+\mu_{F}-\lambda_{-}\right)\left(1+\mu_{I}-\lambda_{-}\right)
\end{gathered}
$$


Inserted into (233) to (235) we obtain, that $\Delta$ becomes

$$
\begin{aligned}
& \frac{1}{\operatorname{det}(D)}\left(1+\mu_{F}-\lambda_{+}\right)\left(1+\mu_{I}-\lambda_{+}\right) \lambda_{+}^{N-1}\left(\left(1+\mu_{F}-\lambda_{-}\right)\left(1+\mu_{I}-\lambda_{-}\right) \delta \beta\right. \\
& \left.-\delta \beta\left(1+\mu_{I}-\lambda_{-}\right)\left(1+\mu_{F}-\tilde{\lambda}\right)\right) \\
& +\frac{1}{\operatorname{det}(D)}\left(1+\mu_{F}-\lambda_{-}\right)\left(1+\mu_{I}-\lambda_{-}\right) \lambda_{-}^{N-1}\left(-\left(1+\mu_{F}-\lambda_{+}\right)\left(1+\mu_{I}-\lambda_{+}\right) \delta \beta\right. \\
& \left.+\delta \beta\left(1+\mu_{I}-\lambda_{+}\right)\left(1+\mu_{F}-\tilde{\lambda}\right)\right) \\
& +\frac{1}{\operatorname{det}(D)}(-\beta)\left(1+\mu_{F}-\tilde{\lambda}\right) \tilde{\lambda}^{N-1}\left(\left(1+\mu_{F}-\lambda_{+}\right)\left(1+\mu_{I}-\lambda_{+}\right)(-\delta)\left(1+\mu_{I}-\lambda_{-}\right)\right. \\
& \left.+\delta\left(1+\mu_{I}-\lambda_{+}\right)\left(1+\mu_{F}-\lambda_{-}\right)\left(1+\mu_{I}-\lambda_{-}\right)\right)
\end{aligned}
$$

Now observe that when $\mu_{F}=\mu_{I}=\mu$

$$
\begin{aligned}
\Delta & =\frac{1}{\operatorname{det}(D)}\left(1+\mu-\lambda_{+}\right)\left(1+\mu-\lambda_{+}\right)\left(1+\mu-\lambda_{-}\right)\left(1+\mu-\lambda_{-}\right) \beta \delta\left(\lambda_{+}^{N-1}-\lambda_{-}^{N-1}\right) \\
& =\beta \frac{(\alpha \delta+\beta \sigma)^{2}}{\left(\lambda_{+}-\lambda_{-}\right)(\alpha \delta+\beta \sigma)^{2}}\left(\lambda_{+}^{N-1}-\lambda_{-}^{N-1}\right)
\end{aligned}
$$

So maximal chemo therapy is optimal in this case as we have seen above. Now compute

$$
\begin{aligned}
\Delta & =\frac{1}{\operatorname{det}(D)} \delta \beta\left(1+\mu_{F}-\lambda_{+}\right)\left(1+\mu_{I}-\lambda_{+}\right)\left(1+\mu_{F}-\lambda_{-}\right)\left(1+\mu_{I}-\lambda_{-}\right)\left(\lambda_{+}^{N-1}-\lambda_{-}^{N-1}\right) \\
+ & \frac{1}{\operatorname{det}(D)} \delta \beta\left(1+\mu_{F}-\lambda_{+}\right)\left(1+\mu_{I}-\lambda_{+}\right)\left(-\left(1+\mu_{I}-\lambda_{-}\right)\right)\left(1+\mu_{F}-\tilde{\lambda}\right)\left(\lambda_{+}^{N-1}-\tilde{\lambda}^{n-1}\right) \\
& +\frac{1}{\operatorname{det}(D)} \delta \beta\left(1+\mu_{F}-\lambda_{-}\right)\left(1+\mu_{I}-\lambda_{-}\right)\left(1+\mu_{I}-\lambda_{+}\right)\left(1+\mu_{F}-\tilde{\lambda}\right)\left(\lambda_{-}^{N-1}-\tilde{\lambda}^{N-1}\right)
\end{aligned}
$$

Notice that for $k=0, \cdots, N-2$

$$
\begin{aligned}
c_{k}= & \frac{1}{\operatorname{det}(D)} \delta \beta\left(1+\mu_{F}-\lambda_{+}\right)\left(1+\mu_{I}-\lambda_{+}\right)\left(1+\mu_{F}-\lambda_{-}\right)\left(1+\mu_{I}-\lambda_{-}\right) \\
& \cdot\left(\lambda_{+}^{N-k-1}-\lambda_{-}^{N-k-1}\right)<0
\end{aligned}
$$

when $\mu=\mu_{F}=\mu_{I}$, due to the assumptions. Observe also that $(246)=0,(247)=$ 0 , when $\mu=\mu_{F}=\mu_{I}$, because then $\tilde{\lambda}=1+\mu$. Now take $\rho>0$ such that

$$
\begin{aligned}
& \mid \frac{1}{\operatorname{det}(D)} \delta \beta\left(1+\mu_{F}-\lambda_{+}\right)\left(1+\mu_{I}-\lambda_{+}\right)\left(-\left(1+\mu_{I}-\lambda_{-}\right)\right)\left(1+\mu_{F}-\tilde{\lambda}\right) \\
& \cdot\left(\lambda_{+}^{N-k-1}-\tilde{\lambda}^{N-k-1}\right) \mid<-\frac{c_{k}}{3}
\end{aligned}
$$

and

$$
\begin{aligned}
& \mid \frac{1}{\operatorname{det}(D)} \delta \beta\left(1+\mu_{F}-\lambda_{-}\right)\left(1+\mu_{I}-\lambda_{-}\right)\left(1+\mu_{I}-\lambda_{+}\right)\left(1+\mu_{F}-\tilde{\lambda}\right) \\
& \cdot\left(\lambda_{-}^{N-k-1}-\tilde{\lambda}^{N-k-1}\right) \mid<-\frac{c_{k}}{3}
\end{aligned}
$$


And finally

$$
\begin{aligned}
& -\frac{1}{\operatorname{det}(D)} \delta \beta\left(1+\mu_{F}-\lambda_{+}\right)\left(1+\mu_{I}-\lambda_{+}\right)\left(1+\mu_{F}-\lambda_{-}\right)\left(1+\mu_{I}-\lambda_{-}\right) \\
& \left(\lambda_{+}^{N-k-1}-\lambda_{-}^{N-k-1}\right)>-\frac{2}{3} c_{k}
\end{aligned}
$$

But then $\Delta<0$, and maximal chemo therapy is optimal. We have used, that the roots of a polynomial depend continuously on the coefficients of the polynomial, see [21]. For $k=N-1$ notice that $(1,0,0) \cdot B=0$. So $u_{N-1}=g_{I}^{0}$ is optimal.

When (ii) define the following $U$ below by computing

$$
\begin{aligned}
& \left(\begin{array}{c}
\left(1+\mu_{F}-a-i b\right)\left(1+\mu_{I}-a-i b\right) \\
-\delta\left(1+\mu_{I}-a-i b\right) \\
-\sigma\left(1+\mu_{F}-a-i b\right)
\end{array}\right) \\
& =\left(\begin{array}{c}
\left(1+\mu_{F}-a\right)\left(1+\mu_{I}-a\right)-b^{2}+i\left(-\left(1+\mu_{I}-a\right) b-\left(1+\mu_{F}-a\right) b\right) \\
-\delta\left(1+\mu_{I}-a\right)+i \delta b \\
-\sigma\left(1+\mu_{F}-a\right)+i \sigma b
\end{array}\right) \\
& =v_{+}=v_{1}+i v_{2}
\end{aligned}
$$

So define $U$ to be

$$
\left(\begin{array}{ccc}
\left(1+\mu_{F}-a\right)\left(1+\mu_{I}-a\right)-b^{2} & -\left(1+\mu_{I}-a\right) b-\left(1+\mu_{F}-a\right) b & -\beta\left(1+\mu_{F}-\tilde{\lambda}\right) \\
-\delta\left(1+\mu_{I}-a\right) & \delta b & \delta \beta \\
-\sigma\left(1+\mu_{F}-a\right) & \sigma b & -\alpha \delta+(1+\gamma-\tilde{\lambda})\left(1+\mu_{F}-\tilde{\lambda}\right)
\end{array}\right)
$$

Then

$$
U^{-1} A U=\left(\begin{array}{ccc}
a & b & 0 \\
-b & a & 0 \\
0 & 0 & \tilde{\lambda}
\end{array}\right)
$$

Define

$$
\begin{aligned}
\tilde{F}(x)= & \left(\left(1+\mu_{F}-a\right)\left(1+\mu_{I}-a\right)-b^{2}\right) x_{1}+\left(-b\left(a-1-\mu_{I}\right)-b\left(a-1-\mu_{F}\right)\right) x_{2} \\
& -\beta\left(1+\mu_{F}-\tilde{\lambda}\right) x_{3}
\end{aligned}
$$

Theorem 3 Suppose (ii) i.e. eigenvalues of $A$ are $a+i b, b \neq 0$. If $N-k-1=2 p+1, k=0, \cdots, N-1$ and

$$
\left\langle e_{1}, A^{N-k-1} B\right\rangle=\frac{\partial \tilde{F}^{\mathrm{T}}}{\partial x}\left(\begin{array}{ccc}
A_{p} & B_{p} & 0 \\
-B_{p} & A_{p} & 0 \\
0 & 0 & \tilde{\lambda}^{2 p+1}
\end{array}\right) U^{-1} B<0
$$

let $v_{k}^{*}=g_{I}^{0}$ and if the reverse inequality holds let $v_{k}^{*}=0$. If $N-k-1=2 p, k=0, \cdots, N-1$ and

$$
\left\langle e_{1}, A^{N-k-1} B\right\rangle=\frac{\partial \tilde{F}^{\mathrm{T}}}{\partial x}\left(\begin{array}{ccc}
C_{p} & D_{p} & 0 \\
-D_{p} & C_{p} & 0 \\
0 & 0 & \tilde{\lambda}^{2 p}
\end{array}\right) U^{-1} B<0
$$

let $v_{k}^{*}=g_{I}^{0}$ and if the reverse inequality holds $v_{k}^{*}=0$. Then $\left(x_{k}^{*}, v_{k}^{*}\right)$ is optim- 
al.

Proof. Define the Hamiltonian

$$
\tilde{H}\left(y_{k}, v_{k}, \zeta_{k}\right)=\zeta_{k}^{\mathrm{T}}\left(U^{-1} A U y_{k}+U^{-1} g+U^{-1} B v_{k}\right)
$$

Then with

$$
\tilde{B}=U^{-1} A U=\left(\begin{array}{ccc}
a & b & 0 \\
-b & a & 0 \\
0 & 0 & \tilde{\lambda}
\end{array}\right)
$$

we find

$$
\zeta_{k}=\left(\tilde{B}^{\mathrm{T}}\right)^{N-k-1} \zeta_{N-1}=\left(\tilde{B}^{\mathrm{T}}\right)^{N-k-1} \frac{\partial \tilde{F}}{\partial x}
$$

So

$$
\frac{\partial \tilde{H}}{\partial v_{k}}=\frac{\partial \tilde{F}^{\mathrm{T}}}{\partial x} \tilde{B}^{N-k-1} U^{-1} B=\frac{\partial \tilde{F}^{\mathrm{T}}}{\partial x}\left(\begin{array}{ccc}
A_{p} & B_{p} & 0 \\
-B_{p} & A_{p} & 0 \\
0 & 0 & \tilde{\lambda}^{2 p+1}
\end{array}\right) U^{-1} B
$$

when $N-k-1=2 p+1, k=0, \cdots, N-1$ and when $N-k-1=2 p, k=0, \cdots, N-1$

$$
\frac{\partial \tilde{F}^{\mathrm{T}}}{\partial x}\left(\begin{array}{ccc}
C_{p} & D_{p} & 0 \\
-D_{p} & C_{p} & 0 \\
0 & 0 & \tilde{\lambda}^{2 p}
\end{array}\right) U^{-1} B
$$

Optimality follows from a computation like (157) to (163).

Theorem 4 Suppose ( $i)$ and the eigenvaues of $A$ are real and distinct. If

$$
\left\langle e_{1}, A^{N-k-1} B\right\rangle=\frac{\partial F^{\mathrm{T}}}{\partial x}\left(\begin{array}{ccc}
\lambda_{+}^{N-k-1} & 0 & 0 \\
0 & \lambda_{-}^{N-k-1} & 0 \\
0 & 0 & \tilde{\lambda}^{N-k-1}
\end{array}\right) D^{-1} B<0
$$

let $v_{k}^{*}=g_{I}^{0}$ and if the reverse inequality holds let $v_{k}^{*}=0$. Then $\left(x_{k}^{*}, v_{k}^{*}\right)$ is optimal.

Proof. Define the Hamiltonian

$$
\tilde{H}\left(y_{k}, v_{k}, \zeta_{k}\right)=\zeta_{k}^{\mathrm{T}}\left(D^{-1} A D y_{k}+D^{-1} g+D^{-1} B v_{k}\right)
$$

Then with

$$
\Lambda=D^{-1} A D
$$

we get

$$
\zeta_{k}=\left(\Lambda^{\mathrm{T}}\right)^{N-k-1} \zeta_{N-1}=\left(\Lambda^{\mathrm{T}}\right)^{N-k-1} \frac{\partial F}{\partial x}
$$

Then the partial derivative of $\tilde{H}$ with respect to $v_{k}$ is

$$
\frac{\partial F^{\mathrm{T}}}{\partial x}\left(\begin{array}{ccc}
\lambda_{+}^{N-k-1} & 0 & 0 \\
0 & \lambda_{-}^{N-k-1} & 0 \\
0 & 0 & \tilde{\lambda}^{N-k-1}
\end{array}\right) D^{-1} B
$$

Optimality follows from a computation like (157) to (163). 


\section{Summary}

In the present paper we applied the discrete Pontryagin Minimal Principle to a discrete model $T$ of cancer growth and the Pontryagin Minimal Principle to an affine vector field that generates $T$. When $\mu=\mu_{F}=\mu_{I}$ and the eigenvalues of $T$ are real and distinct, maximal chemo therapy is optimal for the discrete model, while this is not necessarily so when the eigenvalues of $A$ are $1+\mu, a+i b, b \neq 0,1+\mu>0$.

For the affine vector field that generates $T$, we have proven similar statements, when $\mu=\mu_{F}=\mu_{I}$. Maximal chemo therapy is optimal, when the eigenvalues of $A$ are real, positive and distinct and this is not necessarily so, when there are imaginary eigenvalues. We finally considered what happens in the discrete model, when $\mu_{F} \neq \mu_{I}$. In particular we have derived an optimal strategy to give chemo or immune therapy.

\section{Conflicts of Interest}

The author declares no conflicts of interest regarding the publication of this paper.

\section{References}

[1] Chr, J. (2016) Larsen Models of Cancer Growth. Journal of Applied Mathematics and Computing, 53, 615-643.

[2] Seierstad, A. and Sydsæter, K. (1988) Optimal Control Theory with Economic Applications North Holland.

[3] Goodwin, G. (2005) Constrained Control and Estimation. An Optimization Approach. Springer Verlag, Berlin.

[4] Swierniak, A., Kimmel, M. and Smieja, J. (2009) Mathematical Modelling as a Tool for Planning Anti Cancer Therapy. European Journal of Pharmacology, 625, 108-121. https://doi.org/10.1016/j.ejphar.2009.08.041

[5] Laird, A.K. (1964) Dynamics of Cancer Growth. British Journal of Cancer, 18, 490-502. https://doi.org/10.1038/bjc.1964.55

[6] Adam, J.A. and Bellomo, N. (1997) A Survey of Models for Tumor-Induced Immune System Dynamics. Birkhäuser, Boston.

[7] Geha, R. and Notarangelo, L. (2012) Case Studies in Immunology: A Clinical Companion. Garland Science, Hamden.

[8] Marks, F., Klingmüller, U. and Müller-Decker, K. (2009) Cellular Signal Processing. Garland Science, Hamden.

[9] Molina-Paris, C. and Lythe, G. (2011) Mathematical Models and Immune Cell Biology. Springer Verlag, Berlin. https://doi.org/10.1007/978-1-4419-7725-0

[10] Murphy, K. (2012) Immunobiology. 8th Edition, Garland Science, Hamden.

[11] Rees, R.C. (2014) Tumor Immunology and Immunotherapy. Oxford University Press, Oxford.

[12] Larsen, J.C. (2016) Hopf Bifurcations in Cancer Models. JP Journal of Applied Mathematics, 14, 1-31.

[13] Larsen, J.C. (2017) A Mathematical Model of Adoptive T Cell Therapy. JP Journal of Applied Mathematics, 15, 1-33. 
[14] Larsen, J.C. (2016) Fundamental Concepts in Dynamics.

[15] Larsen, J.C. (2017) The Bistability Theorem in a Cancer Model. International Journal of Biomathematics, 11, Article ID: 1850004.

[16] Larsen, J.C. (2016) The Bistability Theorem in a Model of Metastatic Cancer. Applied Mathematics, 7, 1183-1206. https://doi.org/10.4236/am.2016.710105

[17] Larsen, J.C. (2017) A Study on Multipeutics. Applied Mathematics, 8, 746-773. https://doi.org/10.4236/am.2017.85059

[18] Larsen, J.C. (2017) A Mathematical Model of Immunity. JP Journal of Applied Mathematics.

[19] Larsen, J.C. (2018) Models of Cancer Growth Revisited. Applied Mathematics, 9, Article ID: 84308.

[20] Uspensky (1948) Theory of Equations. McGraw-Hill, New York.

[21] Alexendarian, A. (2013) On Continuous Dependence of Roots of Polynomials on Coefficients. 